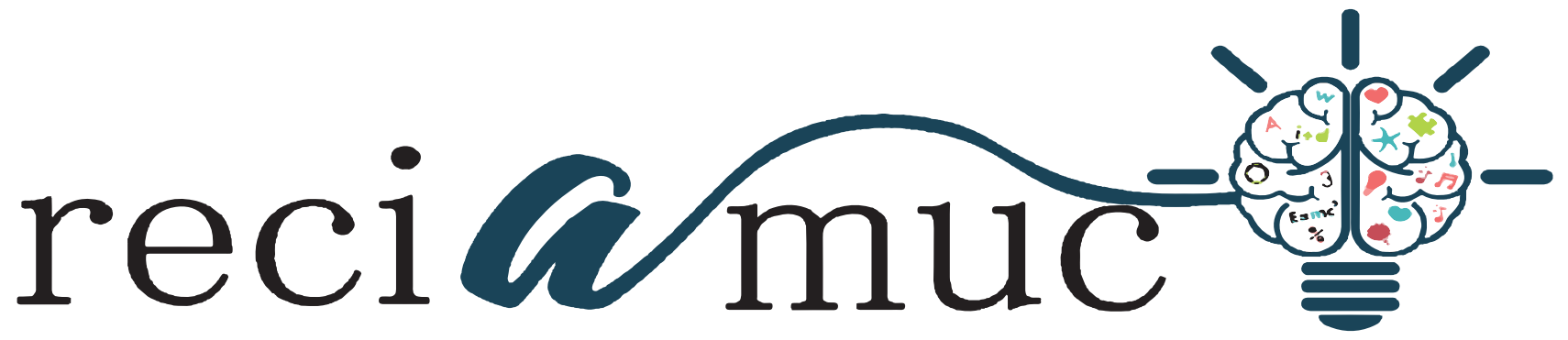

DOl: 10.26820/reciamuc/4.(4).diciembre.2020.125-133

URL: https://reciamuc.com/index.php/RECIAMUC/article/view/579

EDITORIAL: Saberes del Conocimiento

REVISTA: RECIAMUC

ISSN: 2588-0748

TIPO DE INVESTIGACIÓN: Artículo de Revisión

CóDIGo UNESCO: 32 Ciencias Médicas; 3201 Ciencias Clínicas

PAGINAS: 125-133

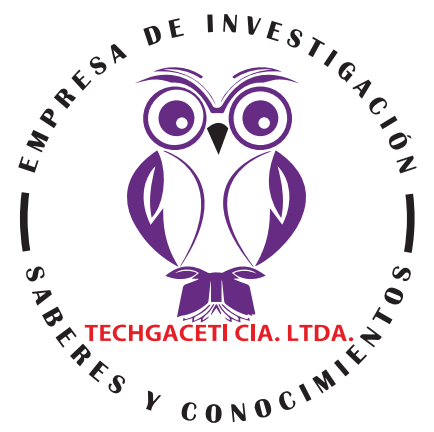

\title{
Síntomas y tratamiento de pacientes diagnosticadas con síndrome de ovario poliquístico
}

Symptoms and treatment of patients diagnosed with polycystic ovary síndrome

Sintomas e tratamento de doentes diagnosticados com síndrome do ovário policístico

\section{Reinaldo Ruiz Rodríguez'; Victoria Katherine Serrano Mera2; Pierina Gardenia Solis Guzmán3; Gilberth Alexander Montes Mendoza ${ }^{4}$}

\section{RECIBIDO: 23/09/2020 ACEPTADO: 17/10/2020 PUBLICADO: 24/12/2020}

1. Especialista de Primer Grado en Medicina General Integral; Especialista de Primer Grado en Obstetricia y Ginecología; Doctor en Medicina; Hospital General Manta; Eurofish S.A; Manta, Ecuador; reinaldoruiz964@gmail. com; https://orcid.org/0000-0003-2410-2987

2. Médica Cirujana; Hospital General Manta; Manta, Ecuador; vyb_88@hotmail.com; https://orcid.org/0000-00021591-2892

3. Médico Cirujano; Médico en Funciones Hospitalarias; Hospital General Manta; damnet_corina@hotmail.com; https://orcid.org/0000-0002-7588-802X

4. Médico Cirujano; Instituto Ecuatoriano de Seguridad Social; Manta, Ecuador; gilberthmontesmendoza@hotmail.com; https://orcid.org/0000-0002-1264-0131

\section{CORRESPONDENCIA}

Reinaldo Ruiz Rodríguez

reinaldoruiz964@gmail.com

\section{Manta, Ecuador}




\section{RESUMEN}

El síndrome de ovario poliquístico (SOP) es uno de los trastornos hormonales más frecuentes en las mujeres en edad reproductiva. Las mujeres con SOP pueden tener períodos menstruales poco frecuentes o prolongados, o niveles excesivos de hormona masculina (andrógeno). Se pueden formar varias acumulaciones pequeñas de líquido (folículos) en los ovarios y estos podrían no liberar óvulos de forma regular. Afecta a un 4-10\% de la población femenina en edad fértil; pero se ha hallado ovario poliquístico (OP) en $24 \%$ de las niñas entre 3 y 18 años. El objetivo fundamental del presente estudio consiste en compendiar las principales manifestaciones clínicas del SOP, así como los principales tratamientos para las pacientes diagnosticadas con dicho síndrome. El modelo de investigación es una revisión de tipo documental bibliográfico. EI SOP puede involucrar una amplia gama de signos y síntomas que pueden variar de una paciente a otra. Entre las principales manifestaciones clínicas se encuentran: anovulación crónica, amenorrea, hiperandrogenismo, alteraciones cutáneas, acné, hirsutismo, resistencia a la insulina, obesidad e incluso debe ser estimado como un factor de riesgo para el desarrollo de la enfermedad cardiovascular. En cuanto al tratamiento, es importante alentar a las pacientes que presentan SOP a realizar actividad física, así como cambiar sus hábitos alimenticios adoptando una alimentación adecuada, lo cual produce beneficios a corto plazo en cuanto a la disminución de la resistencia insulínica y con la prevención de enfermedades crónicas tales como: diabetes mellitus tipo 2 y enfermedad cardiovascular, a largo plazo. Es de vital importancia aumentar los esfuerzos en cuanto a la educación de las pacientes acerca del SOP con la finalidad de que estas lleven a cabo los cambios requeridos en su estilo de vida (dieta y ejercicio) con la finalidad de mejorar esta condición y evitar sus complicaciones.

Palabras clave: Síntomas, Tratamiento, Síndrome, Ovario, Poliquístico.

\section{ABSTRACT}

Polycystic ovary syndrome (PCOS) is one of the most common hormonal disorders in women of reproductive age. Women with PCOS may have infrequent or prolonged menstrual periods, or excessive levels of the male hormone (androgen). Several small collections of fluid (follicles) can form in the ovaries, and the ovaries may not release eggs on a regular basis. It affects $4-10 \%$ of the female population of childbearing age; but polycystic ovary (OP) has been found in $24 \%$ of girls between 3 and 18 years old. The main objective of this study is to summarize the main clinical manifestations of PCOS, as well as the main treatments for patients diagnosed with this syndrome. The research model is a bibliographic documentary type review. PCOS can involve a wide range of signs and symptoms that can vary from one patient to another. Among the main clinical manifestations are: chronic anovulation, amenorrhea, hyperandrogenism, skin alterations, acne, hirsutism, insulin resistance, obesity and it should even be considered as a risk factor for the development of cardiovascular disease. Regarding treatment, it is important to encourage patients with PCOS to engage in physical activity, as well as to change their eating habits by adopting an adequate diet, which produces short-term benefits in terms of reducing insulin resistance and prevention of chronic diseases such as: type 2 diabetes mellitus and cardiovascular disease, long term. It is vitally important to increase efforts regarding the education of patients about PCOS in order for them to carry out the required changes in their lifestyle (diet and exercise) in order to improve this condition and avoid its complications.

Keywords: Symptoms, Treatment, Syndrome, Ovary, Polycystic.

\section{RESUMO}

A síndrome do ovário policístico (PCOS) é uma das perturbações hormonais mais comuns nas mulheres em idade reprodutiva. As mulheres com PCOS podem ter períodos menstruais infrequentes ou prolongados, ou níveis excessivos da hormona masculina (androgénio). Podem formar-se várias pequenas colecções de líquidos (folículos) nos ovários, e os ovários podem não libertar óvulos com regularidade. Afecta 4-10\% da população feminina em idade fértil; mas o ovário policístico (OP) foi encontrado em $24 \%$ das raparigas entre os 3 e 18 anos de idade. O principal objectivo deste estudo é resumir as principais manifestações clínicas de PCOS, bem como os principais tratamentos para pacientes diagnosticados com esta síndrome. O modelo de investigação é uma revisão de tipo documental bibliográfico. A PCOS pode envolver uma vasta gama de sinais e sintomas que podem variar de um paciente para outro. Entre as principais manifestações clínicas estão: anovulação crónica, amenorreia, hiperandrogenismo, alterações cutâneas, acne, hirsutismo, resistência à insulina, obesidade e deve mesmo ser considerado como um factor de risco para o desenvolvimento de doenças cardiovasculares. Relativamente ao tratamento, é importante encorajar os pacientes com PCOS a praticar actividade física, bem como a mudar os seus hábitos alimentares, adoptando uma dieta adequada, que produza benefícios a curto prazo em termos de redução da resistência à insulina e prevenção de doenças crónicas, tais como: diabetes mellitus tipo 2 e doença cardiovascular, a longo prazo. É de vital importância aumentar os esforços no que diz respeito à educação dos pacientes sobre PCOS, a fim de que estes possam realizar as mudanças necessárias no seu estilo de vida (dieta e exercício), a fim de melhorar esta condição e evitar as suas complicações.

Palavras-chave: Sintomas, Tratamento, Síndrome, Ovário, Policístico. 


\section{Introducción}

El síndrome de ovario poliquístico (SOP) es uno de los trastornos hormonales más frecuentes en las mujeres en edad reproductiva. "Las mujeres con SOP pueden tener períodos menstruales poco frecuentes o prolongados, o niveles excesivos de hormona masculina (andrógeno). Se pueden formar varias acumulaciones pequeñas de líquido (folículos) en los ovarios y estos podrían no liberar óvulos de forma regular". (Clínica Mayo, 2020)

Calletano, citado por Suarez, Borja, Vela, \& Ontaneda, (2019) refiere en cuanto a los antecedentes del SOP lo siguiente:

En 1935, Stein y Leventhal denominaron síndrome de ovarios poliquísticos (SOP) a un cuadro clínico caracterizado por la presencia de ovarios con pequeños quistes, amenorrea, hirsutismo y obesidad. Inicialmente fue llamado síndrome de Stein Leventhal, y en la actualidad como hiperandrogenismo ovárico funcional. (p. 973)

EI SOP está ligado a cambios en los niveles hormonales que le dificultan a los ovarios la liberación de óvulos maduros. Las razones para estos cambios no son claras. Las hormonas afectadas son:

- Los estrógenos y la progesterona.

- Los andrógenos. (Enciclopedia Médica ADAM, 2021)

"El síndrome de ovario poliquístico (SOP) ocurre en 5 a 10\% de las mujeres. En los Estados Unidos, es la causa más común de infertilidad". (Pinkerton, 2019)

Chiliquinga, Aguirre, Agudo, Chú, \& Cuenca, (2017) acotan al respecto que:

Afecta a un 4-10 \% de la población femenina en edad fértil; pero se ha hallado ovario poliquístico (OP) en $24 \%$ de las niñas entre 3 y 18 años. Las adolescentes que acuden a consulta por trastornos menstruales, hirsutismo (presente hasta en el $80 \%$ de los casos), obesidad o la presencia ultrasono- gráfica accidental de SOP, muchas veces son valoradas por separado entre endocrinólogos, ginecólogos o internistas sin la debida visión integral de riesgo vascular, en respuesta a la insulinoresistencia (IR) piedra angular del síndrome metabólico (SM) y presente también en el SOP. (p. 174)

Con respecto a las complicaciones asociadas al SOP, Del Castillo, Martínez, \& Del Castillo, (2014) explican que pueden ser muy variadas y entre las principales se encuentran: cambios en el humor, hígado graso no alcohólico, aumento de riesgo car $\neg-$ diovascular, aumento del riesgo del cáncer de endo $\neg$ metrio y otros. (p. 2)

Un diagnóstico temprano y preciso es fundamental para controlar este síndrome y las complicaciones que a este se relacionan, en virtud de lo cual el conocimiento de sus síntomas o manifestaciones clínicas es fundamental, tanto por parte del paciente para que acuda a la consulta como por parte del médico tratante para precisar el diagnóstico y el tratamiento más eficaz.

El objetivo fundamental del presente estudio consiste en compendiar las principales manifestaciones clínicas del SOP, así como los principales tratamientos para las pacientes diagnosticadas con dicho síndrome.

\section{Materiales y métodos}

Para el desarrollo del presente estudio fueron ubicados una serie de archivos digitalizados, cuya búsqueda de contenidos científicos se llevó a cabo durante el mes de diciembre de 2020 y primeros días de enero 2021. En virtud de lo cual la presente investigación se clasifica como de tipo documental bibliográfico.

Se formularon ecuaciones de búsqueda, con palabras clave, operadores lógicos y booleanos, en determinadas bases de datos y/o buscadores especializados, tales como: SciELO, Researchgate, entre otros. Asimismo, se usaron páginas web relacionadas con el área de la salud, con amplio 
reconocimiento científico a nivel nacional e internacional, entre las que destacamos: Manuales MSD, Clínica Mayo, MedlinePlus y Sociedad Americana de Medicina Reproductiva.

Algunas de las formulaciones y descriptores utilizados con los que se obtuvieron mejores resultados fueron los siguientes: "síndrome de ovario poliquístico"; "manifestaciones clínicas del síndrome de ovario poliquístico"; "tratamiento del síndrome de ovario poliquístico". La información obtenida fue filtrada bajo los siguientes criterios: idioma (español); disponibilidad del contenido (completo); periodo de publicación (2012-2021, con excepción de un artículo del 2003 de contenido teórico vigente), tipo de estudio: revisiones sistemáticas, de cohorte y de casos o de controles, informe de casos; y, clase de material bibliográfico (consensos, manuales, ensayos, tesis de grado, posgrado o doctorado, informes y otras clases de contenidos).

Por último, fueron desestimados aquellos contenidos repetidos (duplicados), editoriales, anotaciones académicas y otros tipos de materiales bibliográficos de escaso valor científico, con bajo nivel de evidencia o aportado por tratadistas sin acreditación en el área de la salud o medicina.

\section{Resultados}

Síntomas o manifestaciones clínicas de las pacientes con Síndrome de Ovario Poliquísticos

Para Vallecillo Torres, (2012) el cuadro clínico del SOP resulta sumamente variado, por cuanto la manifestación de sus diversos síntomas depende de cada caso en particular. Este particular sigue generando polémica entre los autores dedicados al estudio de este trastorno, no obstante, se entre los más significativos y de mayor impacto sobre este grupo de pacientes se consideran los siguientes: anovulación crónica, amenorrea, hiperandrogenismo, alteraciones cutáneas, acné, hirsutismo, resistencia a la insu- lina, obesidad e incluso debe ser estimado como un factor de riesgo para el desarrollo de la enfermedad cardiovascular. (p. 432)

Asimismo, es importante destacar que la mayoría de los datos clínicos que aparecen en pacientes con SOP pueden formar parte de otras patologías que no necesariamente se encuentren asociadas con alguna disfunción hormonal, en virtud de lo cual, la asociación de síntomas y signos y no los hechos aislados y siempre deben sugerir el diagnóstico. Lo severo o rápido en su evolución debe ser motivo de alerta para la realización de un diagnóstico diferencial más extenso. Para Vargas, Sánchez, Herrera, \& Vargas, (2003) las manifestaciones más frecuentes son las siguientes:

- Acné. Aparece frecuentemente en las adolescentes y generalmente para los 15 años de edad el 50\% de ellas lo presentará. Su permanencia de los 20 años en adelante debe considerarse sospechosa. Es imperativo interrogar acerca de irregularidades menstruales, hirsutismo o cualquier otro dato de hiperandrogenismo. En estudios realizados en mujeres con acné se ha encontrado datos de SOP hasta en el $45 \%$ de los casos.

- Hirsutismo: Se define como el crecimiento excesivo del vello corporal terminal en mujeres, en áreas anatómicas donde el desarrollo de los folículos depende de la estimulación androgénica: Tercio proximal en cara interna de muslos, abdomen, pecho, parte baja de la espalda y cara entre otros. El grado y la severidad se evalúa de acuerdo a la escala de Ferriman-Galwey.

- Virilización: La aparición de hipertrofia clitorídea, amenorrea prolongada, aumento de la musculatura, atrofia de los senos, hirsutismo severo y habitus masculino obliga a descartar la presencia de hiperplasia adrenal, hipertecosis o tumores ováricos o adrenales.

- Irregularidad menstrual y anovulación: 
Las mujeres con SOP presentan grados variables de disfunción ovulatoria, manifestada como oligomenorrea, dismenorrea y amenorrea. Incluso, frecuentemente presentan infertilidad $(17.5 \%$ vs $1.3 \%$ en normales). La relación entre el exceso de insulina y la anovulación se atribuye en primer lugar a la hiperandrogenemia y en segundo a la estimulación del inhibidor 1 del activador del plasminógeno (PAI-1).

- Acanthosis Nigricans: Se trata de una hiperplasia hiperpigmentada de la piel, la cual aparece predominantemente en el cuello y en pliegues cutáneos como axilas y codos. La importancia de su detección radica en que su presencia correlaciona significativamente con los estados de resistencia a la insulina e hiperinsulinemia compensatoria. La presentan cerca del $40 \%$ de las adolescentes nativas americanas, $13 \%$ de las africo-americanas, $6 \%$ de las hispanas y menos del $1 \%$ de las caucásicas. (p. 194, 195)

\section{Tratamiento del Síndrome de Ovario Poli- quístico}

Para Muñoz Calvo, (2016) los objetivos del tratamiento del SOP son los siguientes:

1. Alcanzar la ovulación

2. Normalizar los ciclos menstruales

3. Disminuir y si es posible eliminar el hirsutismo y el acné

4. Obtener una pérdida de peso aceptable

5. Tratar la dislipemia y la hiperglucemia para disminuir el riesgo de ECV. (p. 25)

El tratamiento se puede dividir en no farmacológico y farmacológico:

- Tratamiento no farmacológico.

- Ejercicio físico y pérdida de peso.

Dado que el SOP se asocia a obesidad y sobrepeso en una ele $\neg$ vada proporción de casos, se recomienda pérdida de peso, tanto aumentando el ejercicio físico como con dieta hipocalórica:

Se recomienda ejercicio físico en el manejo de las complicaciones de obesidad y sobrepeso de estas pacientes. Aunque no existen estudios randomiza $\neg$ dos que justifiquen esta práctica, su uso en combi $\neg$ nación con el resto de medidas higiénico-dietéticas mejora la pérdida de peso, reducen la incidencia de desarrollo de diabetes y mejoran el resto de factores de riesgo cardiovascular. (Del Castillo, Martínez, \& Del Castillo, 2014, p. 8)

Chiliquinga, Aguirre, Agudo, Chú, \& Cuenca, (2017) refieren al respecto que:

El tratamiento no farmacológico debe estar basado en la reducción del peso en 5 \% del peso inicial, la pérdida de grasa abdominal mejora el perfil endocrino y aumenta la probabilidad de la ovulación y el embarazo. Las dietas serán bajas en calorías y en grasa, junto con un compuesto con aminoácidos, extractos y antioxidantes. Estas disminuyen la resistencia a la insulina y adicionan vitamina D para ayudar con la fertilidad e incorporar la actividad física por lo menos 90 minutos de actividad aeróbica a la semana. Si la pérdida de peso espontánea no se puede lograr con dieta y ejercicio, puede ofrecerse la cirugía bariátrica. Igualmente, El tratamiento integral también debe considerar medidas como consejería, apoyo psicológico, técnicas de meditación, relajación, educación preventiva y participación en grupos de ayuda y acompañamiento familiar. La medicina alternativa incluye muchas modalidades, como la kinesiología, la fitoterapia, la homeopatía, la reflexología, la acupresión, la acupuntura y la terapia de masaje. La acupuntura es la modalidad más común, pues parece tener beneficios para los que sufren del SO al ayudar a regular y gestionar sus períodos. Sin embargo, también se ha demostrado su utilidad para ayudar en la pérdida de peso, dolores de cabeza y mejorar los estados de ánimo. (p. 178) 
Fonseca Villanea, (2018) acota acera del tretamiento no farmacológico que, uno de los síntomas que más afecta a las pacientes quienes padecen SOP es el hirsutismo, en el que el tratamiento cosmético con rasurado, depilación con seca o láser puede ser beneficioso en casos leves. Como muchas, esta terapia no se encuentra exenta de efectos secundarios, como la dermatitis por contacto, foliculitis hasta sepsis. La Eflornitina se usa vía tópica como una alternativa en el tratamiento cosmético. "Estudios preliminares indican que puede prolongar los periodos de remisión. La Eflornitina solo se ha estudiado para hirsutismo facial y evita los efectos secundarios de los tratamientos sistémicos. De no ser efectiva la terapia cosmética se debe plantear tratamiento antiandrogénico". (p. 12)

\section{Tratamiento farmacológico}

\section{Anticonceptivos hormonales (AHC)}

En cuanto al tratamiento con anticonceptivos orales, Muñoz Calvo, (2016) refiere lo siguiente:

La combinación de etinilestradiol (EE) y progesterona suprime la producción ovárica de andrógenos, incrementa los niveles de SHBG (La globulina fijadora de hormonas sexuales) y disminuye las concentraciones de testosterona libre. La progesterona evita la hiperplasia endometrial. Una dosis de 30 mcg de EE por día es suficiente para suprimir la actividad folicular ovárica y disminuir los síntomas de HA. Se utilizan AHC de tercera o cuarta generación, que contengan progestágenos de acción antiandrogénica como el acetato de ciproterona y la drosperinona (derivado de la espironolactona). El acetato de ciproterona parece ser más eficaz en el tratamiento del hirsutismo, disminuyendo los niveles de testosterona y delta-4-androstendiona y normalizando el ratio $\mathrm{FSH} / \mathrm{LH}$, pero a su vez, empeora el estado de resistencia a la insulina y tiene un efecto negativo en el perfil lipídico, produciendo aumento de triglicéridos. Asimismo, la drospirenona es eficaz en la mejoría de la rela- ción cintura/cadera. Entre los 6 y 9 meses de su utilización se puede encontrar mejoría del hirsutismo y se recomienda utilizarlo al menos dos años. (p. 25, 26)

\section{Sensibilizadores a la insulina}

Para el tratamiento contra la resistencia a la insulina en pacientes con SOP, la Sociedad Americana de Medicina Reproductiva, (2012) recomienda las siguientes opciones:

Es muy importante bajar de peso, mejorar la nutrición y hacer ejercicio. El cambio de conducta debería ser la primera línea de tratamiento en el caso de una mujer obesa con SOP. Los fármacos aprobados por la Administración de Drogas y Alimentos (Food and Drug Administration, FDA) para el tratamiento de la diabetes tipo 2 parecen prometedores para el tratamiento del SOP. Estos fármacos, conocidos como agentes sensibilizadores a la insulina, mejoran la respuesta del organismo a la insulina. El mejor agente sensibilizador a la insulina que se ha estudiado y que se encuentra disponible en los Estados Unidos para tratar a las mujeres con SOP es la metformina. La metformina reduce los niveles circulantes de insulina y de andrógeno (hormona masculina) y restablece la ovulación normal en algunas mujeres con SOP. Uno de los efectos secundarios comunes es la irritación gastrointestinal, especialmente con diarrea y náuseas. Estos síntomas suelen mejorar después de unas pocas semanas y se pueden reducir aumentando gradualmente la dosis. Un efecto secundario poco frecuente pero serio de la metformina es la acidosis láctica: una acumulación de ácido en el torrente sanguíneo causada por el metabolismo ineficiente. Puede provocar respiración profunda y rápida, vómitos, dolor abdominal, letargo y alteraciones del ritmo cardíaco. No se recomienda la metformina en pacientes con nefropatía, neumopatía, hepatopatía o cardiopatía. La metformina debe suspenderse temporalmente antes de realizar una cirugía o una radiografía en la que se administra contraste por vía intrave- 
nosa a causa del alto riesgo de acidosis láctica. Del mismo modo, ninguna persona que tenga una alimentación con bajo contenido de carbohidratos debe recibir metformina. Se ha demostrado que la metformina y otras tiazolidinedionas reducen el hiperandrogenismo (el incremento de los niveles de hormonas masculinas y el crecimiento excesivo de vello) y restablecen la ovulación en algunas pacientes con SOP. La pioglitazona no se utiliza comúnmente en las pacientes con SOP y, si es necesario utilizarla, un médico experimentado debe realizar su seguimiento.

\section{Bloqueantes del receptor de andrógenos}

La espironolactona es un antiandrógeno utilizado en el tratamiento del HA, especialmente en el hirsutismo. Varios estudios en adolescentes con SOP demuestran que produce una mejoría clínica del HA y de las irregularidades menstruales, pero no de las alteraciones metabólicas. Tanto la espironolactona como el finasteride está fuera de ficha técnica. La flutamida, no es aconsejable por el riesgo de hepatoxicidad hepática además de no tener indicación pediátrica. (Muñoz Calvo, 2016, 27)

\section{Tratamiento del hirsutismo}

Fung, (2016) explica que el hirsutismo es el signo clínico de mayor importancia de hiperandrogenismo y se encuentra presente en aproximadamente $70 \%$ de las pacientes con SOP. "El hirsutismo se define como el crecimiento excesivo de pelo terminal (vello largo, grueso, pigmentado) en la mujer siguiendo un patrón masculino de distribución, en zonas andrógenodependientes". (p. s27)

El tratamiento de primera línea para el hirsutismo no está totalmente establecido. Además, muchas veces los resultados no son tan efectivos como la mujer desea. En cuanto a las opciones de tratamiento Pérez \& Maroto, (2018) refieren lo siguiente:

- ACO: Ninguno ha sido aprobado para el tratamiento del hirsutismo; sin embargo, los que contienen drospirenona parecen tener un ligero beneficio en estas mujeres. También se ha probado la combinación de un ACO sin drospirenona con espironolactona.

- Anti androgénicos: Son antagonistas de la unión de la testosterona y otros andrógenos con su receptor. Son levemente beneficiosos y deben ser utilizados con anticoncepción por su riesgo de teratogenicidad.

- Espironolactona: Además de ser un antagonista de la aldosterona, esta droga se une al receptor androgénico, por lo que funciona como un antagonista competitivo. La dosis utilizada es de 25- 100 mg BID, siempre se deben monitorizar los niveles de potasio, por el riesgo de hiperkalemia que esta droga supone, sobre todo en mujeres con daño renal.

- Eflornitina: Es un inhibidor de la enzima ornitina descarboxilasa, de uso tópico. Se utiliza para las pacientes con clínica de hirsutismo a nivel facial. Se aplica dos veces al día en las áreas faciales afectadas. Este tratamiento ya ha sido aprobado por la Food and Drug Administration (FDA). (nivel A). (p. 7, 8)

\section{Conclusiones}

El Síndrome de Ovario Poliquístico (SOP) es un trastorno complejo y controversial, por sus múltiples y variados signos y síntomas, y su manejo, que afecta a un gran número de pacientes femeninas en edad reproductiva.

Un diagnóstico adecuado y oportuno, así como su posterior tratamiento específico son factores que incrementarán las posibilidades de control de este síndrome, así como de sus complicaciones, aumentar la capacidad de concebir en las pacientes que así lo deseen, el alivio de sus síntomas y minimizará o evitará los efectos secundarios que puedan presentarse a largo plazo. 
El SOP puede involucrar una amplia gama de signos y síntomas que pueden variar de una paciente a otra. En cuanto al tratamiento, es importante alentar a las pacientes que presentan SOP a realizar actividad física, así como cambiar sus hábitos alimenticios adoptando una alimentación adecuada, lo cual produce beneficios a corto plazo en cuanto a la disminución de la resistencia insulínica y con la prevención de enfermedades crónicas tales como: diabetes mellitus tipo 2 y enfermedad cardiovascular, a largo plazo.

Es de vital importancia aumentar los esfuerzos en cuanto a la educación de las pacientes acerca del SOP con la finalidad de que estas lleven a cabo los cambios requeridos en su estilo de vida (dieta y ejercicio) con la finalidad de mejorar esta condición y evitar sus complicaciones.

\section{Bibliografía}

Chiliquinga, S., Aguirre, R., Agudo, M., Chú, Á., \& Cuenca, S. (2017). Criterios diagnósticos y tratamiento integral terapéutico del síndrome de ovarios poliquísticos. Revista Cubana de Ginecología y Obstetricia, 43(3), 173-181. Recuperado el 10 de Diciembre de 2020, de http://scielo.sld.cu/pdf/gin/ v43n3/gin18317.pdf

Clínica Mayo. (03 de Octubre de 2020). Clínica Mayo. Recuperado el 02 de Diciembre de 2020, de https://www.mayoclinic.org/es-es/diseases-conditions/pcos/symptoms-causes/syc-20353439

Del Castillo, F., Martínez, A., \& Del Castillo, R. (2014). Guía de práctica clínica de síndrome de ovario poliquístico. Archivos de Medicina, 10(2:3), 1-14. doi:10.3823/121

Enciclopedia Médica ADAM. (05 de Enero de 2021). MedlinePlus. Recuperado el 06 de Enero de 2021, de https://medlineplus.gov/spanish/ency/article/000369.htm

Fonseca Villanea, C. (Junio de 2018). Síndrome de ovario poliquístico. Revista Médica Sinergia, 3(6), 9-15. doi:https://doi.org/10.31434/rms.v3i6.130

Fung, L. (2016). Diagnóstico clínico y bioquímico del síndrome de ovario. Revista de Ginecología y Obstetricia Venezolana, 76(1), S25-S34. Recuperado el 19 de Diciembre de 2020, de http://ve.scielo.org/pdf/og/v76s1/art06.pdf
Muñoz Calvo, M. (2016). Síndrome de Ovario Poliquístico (SOP). Revista de Formación Continuada de la 20 Sociedad Española de Medicina de la Adolescencia, 4(2), 20-31. Recuperado el 16 de Diciembre de 2020, de https://www.adolescenciasema.org/ficheros/REVISTA\%20ADOLESCERE/ vol4num2-2016/20_sindrome_de_ovario_poliquistico_sop.pdf

Pérez, J. M., \& Maroto, K. E. (Marzo de 2018). Síndrome de Ovarios Poliquísticos. Medicina Legal de Costa Rica Edición Virtual, 35(1), 1-8. Recuperado el 05 de Enero de 2021, de https://www.scielo.sa. cr/pdf/mlcr/v35n1/1409-0015-mlcr-35-01-94.pdf

Pinkerton, J. V. (2019). Manuales MSD. Recuperado el 03 de Diciembre de 2020, de https://www. msdmanuals.com/es/professional/ginecolog\%C3\%ADa-y-obstetricia/anomal\%C3\%ADas-menstruales/s\%C3\%ADndrome-del-ovario-poliqu\%C3\%ADstico-sop

Sociedad Americana de Medicina Reproductiva. (2012). Sociedad Americana de Medicina Reproductiva. Recuperado el 18 de Diciembre de 2020, de http://txfertility.com/wp-content/ uploads/2013/10/Agentes-sensibilizantes-a-la-insulina-y-s\%C3\%ADndrome-de-ovario-poliqu\%C3\%ADstico.pdf

Suarez, B. H., Borja, P. E., Vela, M. A., \& Ontaneda, C. F. (2019). Diagnóstico y manejo del síndrome de ovario poliquístico. Revista Científica Mundo de la Investigación y el Conocimiento (Recimundo), 3(3), 970-1004. Recuperado el 05 de Diciembre de 2020, de https://dialnet.unirioja.es/descarga/ articulo/7116499.pdf

Vallecillo Torres, A. (2012). Diagnóstico de Síndrome de Ovario Poliquístico. Revista Médica de Costa Rica y Centroamérica, 69(603), 431-434. Recuperado el 20 de Diciembre de 2020, de https://www. medigraphic.com/pdfs/revmedcoscen/rmc-2012/ rmc124t.pdf

Vargas, M. A., Sánchez, G., Herrera, J., \& Vargas, L. (2003). Síndrome de ovarios poliquísticos: abordaje diagnóstico y terapéutico. Revista Biomed, 14, 191-203. Recuperado el 18 de Diciembre de 2020, de https://www.researchgate. net/publication/267549197_Sindrome_de_ovarios_poliquisticos_abordaje_diagnostico_y_terapeutico/fulltext/5457389e0cf2bccc490f4db4/ Sindrome-de-ovarios-poliquisticos-abordaje-diagnostico-y-terapeutico.pdf?origin=publication_detail 


\section{CITAR ESTE ARTICULO:}

Ruiz Rodríguez, R., Serrano Mera, V. K., Solis Guzmán, P. G., \& Montes Mendoza, G. A. (2021). Síntomas y tratamiento de pacientes diagnosticadas con síndrome de ovario poliquístico. RECIAMUC, 4(4 (esp), 125-133. https://doi. org/10.26820/reciamuc/4.(4).diciembre.2020.125-133 